\title{
ELECTRICIDAD Y CRECIMIENTO ECONOMICO. LOS INICIOS DE LA ELECTRICIDAD EN ESPAÑA *
}

FRANCESCA ANTOLIN

Universidad Autónoma de Barcelona

En las tres primeras décadas del siglo xx la economía de los países industriales avanzados experimentó cambios profundos, al tiempo que conseguía tasas de crecimiento relativamente altas. La introducción de la electricidad y su pronta difusión han sido consideradas fundamentales para explicar el crecimiento de este período. Pero el papel exacto de esta nueva forma de energía en el progreso industrial y en el crecimiento económico está sujeto a cualificaciones, incluso a controversia. El objeto de este trabajo es ofrecer un primer contraste de hipótesis respecto a las causas económicas que marcan el ritmo y el grado de extensión de la electricidad en el sector industrial español -con énfasis especial en el caso del País Vasco-. Con este fin, me refiero en primer lugar a las contribuciones de varios autores respecto a la conexión entre electricidad y crecimiento y a los mecanismos que activan el proceso de electrificación. En particular, destaco la hipótesis según la cual el grado de electrificación, y la velocidad a la que ésta se produce, puede ser más debida a consideraciones de precios relativos entre tipos de energía alternativos y otros factores de producción que a ventajas absolutas de carácter técnico. En una segunda parte, y tras reunir la evidencia empírica pertinente, compruebo cómo, tanto en el conjunto español como en el caso vasco, coincidiendo con la presencia de unos mecanismos incentivadores muy débiles, se da un proceso de electrificación lento y poco profundo. Además de reforzar la tesis de Woolf (1984), la experiencia analizada es coherente con lo que ya sabemos respecto al comportamiento en España del sector energético (Sudrià, 1987) e industrial (Carreras, 1987). En nuestro país no parece darse la conexión automática apuntada por Devine (1983) entre electricidad y crecimiento económico.

* Quiero agradecer la extrema amabilidad y complacencia de Luis de León, de la so ciedad Iberduero, al proporcionarme información imprescindible para el estudio del sector eléctrico español, del que este artículo es una primera aproximación. Estoy en deuda tam. Maluquer, A Carreras y S. Coll por sus valiosas sugerencias y comentarios a este trabajo. 
Algunas tesis sobre la contribución

de la electricidad al crecimiento económico

Landes (1969) califica de Segunda Revolución Industrial al proceso de profunda renovación económica que se produce en los años correspondientes al primer tercio de siglo. En ellos, una nueva forma de energía, la electricidad, y su pareja, el motor eléctrico, tuvieron un protagonismo parecido al detentado por el vapor y las máquinas textiles un siglo antes. Según este autor, las contribuciones más notables de la electricidad, la más flexible de todas las formas de energía, al desarrollo económico consistieron en: a) incrementar la oferta de energía primaria, al permitir utilizar la energía cinética del agua sin imponer restricciones a la localización de las empresas; $b$ ) ahorrar combustible, al ser las técnicas de producción y transmisión de electricidad más eficientes que las de energías alternativas; $c$ ) como consecuencia de las dos anteriores, poner a disposición de los usuarios energía abundante a un menor coste, $\mathrm{y} d$ ) finalmente, permitir cambios profundos en las técnicas y métodos de producción para una amplia gama de actividades industriales, dando paso además al nacimiento de nuevas industrias (de producción de electricidad, de equipo y maquinaria eléctrica, electrodomésticos, etc.).

Posteriormente, y basándose en la experiencia de países concretos, otros autores han formulado propuestas más precisas sobre la relación entre electrificación y crecimiento económico. Devine describe las claves del proceso de adopción de la electricidad en Estados Unidos y relaciona dicho proceso con el crecimiento de la productividad de la economía americana. Siguiendo las consideraciones hechas por ingenieros $\mathrm{y}$ empresarios contemporáneos a la electrificación, distingue entre dos tipos de efecto de la electricidad sobre el proceso productivo:

a) Aborro en costes directos. La electricidad permitió reducir la cantidad de energía necesaria para accionar las máquinas gracias a la adopción del sistema de unit drive, consistente en que cada proceso mecánico disponga de un motor individualizado, de potencia y velocidad específicas para cada función. El unit drive se introdujo en 1890 y se convirtió en el tipo de instalación predominante desde el final de la Primera Guerra Mundial. Con este sistema sólo se consume energía cuando la máquina funciona, por lo que, en principio, se introducen importantes ahorros respecto a la técnica anterior, consistente en disponer de un único motor que acciona simultáneamente todas las máquinas conectadas a él por un sistema de ejes y poleas. Sin embargo, el coste de la energía propiamente dicha es sólo una parte de los costes de una opción energética. También lo son los de capital (maquinaria), trabajo y materiales asociados a dicha opción. El sistema de unit drive 
genera ahorros en los demás factores, pero encarece el coste de capital, puesto que la potencia instalada en plantas que utilizan esta técnica se multiplica entre 5 y 7 respecto a plantas con sistemas de transmisión de energía utilizadas anteriormente. Teniendo en cuenta los distintos componentes de coste asociados àl consumo de energía en la experiencia analizada por Devine, éste concluye que el unit drive podía ser un poco más barato que los sistemas precedentes, pero no necesariamente.

b) Beneficios indirectos. A principio de siglo, los promotores de la electricidad afirmaban que, a menudo, de la adopción de esta forma de energía se deducían incrementos importantes de la producción de un 20 a un 30 por 100, incluso manteniendo la misma dotación de los demás factores. Dicho crecimiento en la productividad se producía porque con el unit drive se incrementaba el ritmo de la producción. La electricidad permitía una total flexibilidad en el diseño de los espacios industriales. Las máquinas podían colocarse en la secuencia natural de los procesos de producción, y así se ahorraba en tiempo y en costes de desplazar materiales dentro de la fábrica. Se ganaba en potencia y control sobre las máquinas, con lo que aumentó la velocidad de las operaciones. Mejoraron las condiciones de trabajo en términos de luz, ventilación, ruido y limpieza. Resultó menos costoso expandir las plantas industriales. Coincidiendo con la opinión de los contemporáneos al inicio de la industria eléctrica, Devine opina que la mayor contribución de la electricidad, y la razón por la que fue adoptada, reside en su capacidad de incrementar la productividad industrial. El atractivo de la electricidad no sería, pues, su supuesto bajo coste, sino su versatilidad, que permitiría revolucionar los métodos de producción. Para probar su hipótesis analiza el comportamiento de la ratio consumo de energía/producto interior bruto (PNB) en el intervalo 1880-1950. Comprueba cómo desde 1920, fecha en que la electricidad se hizo predominante, el consumo de energía por unidad de PNB fue disminuyendo consistentemente, mientras crecían la productividad del trabajo y del capital.

A mi entender, el razonamiento de Devine presenta algunos problemas. Al relacionar de forma exclusiva el avance de la productividad con el consumo de energía, fuerza la implicación de que «electricidad» es sinónimo de «crecimiento económico». De hecho, el crecimiento de la productividad es un fenómeno complejo que depende de muchos factores (Marshall, 1982). Lo único que demuestra Devine es una coincidencia temporal entre ciertos comportamientos del sector energético con los de la productividad. Para poder aislar los distintos efectos de la introducción de la electricidad, la medida en que ésta es, simplemente, más productiva, y la medida en que su intro- 
ducción se deba, en cambio, a su bajo coste, conviene un análisis más sofisticado. Esto es lo que se propone Woolf (1984).

También este autor coincide en señalar la importancia de la introducción de la electricidad sobre la economía americana. Supuso poder disponer de una oferta de energía casi ilimitada a un coste marginal constante, si no decreciente. Permitió, además, una gran libertad en el diseño de las plantas industriales, con lo que dejó la puerta abierta a la innovación en las técnicas de producción. Pero el objetivo más concreto de su trabajo es intentar demostrar que «una porción importante de las mejoras habidas en la productividad durante el período 1900-1930 pueden atribuirse a la electrificación». Para ello estima una función de producción translog en la que incluye dos inputs energéticos, electricidad y carbón, además del capital y trabajo, clásicos en este tipo de modelos, utilizando datos de los censos industriales de Estados Unidos para el período 1909-1929. Los resultados empíricos no son concluyentes. A pesar de su tesis inicial, lo único que se desprende de su análisis sector a sector es que, "para cada uno de ellos, la combinación carbón-electricidad varía exclusivamente en función del cambio en los precios relativos de dichos inputs» y que "no se aprecia en el período estudiado ningún sesgo en el cambio tecnológico que tienda a favorecer las innovaciones que utilizan electricidad». De acuerdo con el modelo de Woolf, la electricificación se explica como combinación de varios efectos ligados a un cambio de precios relativos. En Estados Unidos, y en el intervalo 1910-1929, el precio de la electricidad se redujo en un 50 por 100, mientras que el del carbón se multiplicaba por 3 y los salarios por más de 2. Este cambio en los precios relativos de los factores, por una parte - al cambiar la pendiente de las isocostes-, favoreció la sustitución de los inputs caros por los baratos (primer estímulo para la adopción de la electricidad). Por otra, siguiendo a Hicks (1932), dicho cambio hizo obsoletas las viejas técnicas industriales y dio paso a nuevas técnicas de producción -alteración de las isocuantassesgadas en la dirección de favorecer el uso de los inputs más baratos (maquinaria y electricidad) y el ahorro de los caros (carbón y mano de obra) [segundo estímulo para la adopción de la electricidad]. La nueva tecnología fue la responsable del cambio en la productividad total de los factores. Su desarrollo sacó provecho de la versatilidad de la electricidad, porque económicamente interesaba utilizarla con mayor intensidad. Si no se hubiera aprendido a utilizar la electricidad, o de haber resultado ésta más cara que el carbón, la tecnología, respondiendo a una situación distinta en los precios relativos, se hubiera desarrollado en otra dirección. Así, pues, aunque la disponibilidad de la nueva técnica acabó resultando crucial, el estímulo inicial más importante sugerido por los datos, en el caso americano, parece ser el económico puro (cambios en los precios relativos), más que las ventajas 
económicas del cambio técnico que se desarrollaría en respuesta a la nueva situación de precios.

\section{Precios relativos de factores y electrificación en España}

A la luz de las tesis utilizadas en la literatura que acabo de describir, parece pertinente preguntarse si la electrificación en España recibió estímulos análogos a los que pudieron desencadenarla en los EE. UU., y por la intensidad de dichos estímulos. No recurriré a un análisis econométrico, entre otras razones porque se carece de datos apropiados sobre la distribución del consumo de inputs energéticos y la formación de capital por sectores industriales, necesarios para la estimación del modelo. Lo que haré será establecer que los precios de los factores, cuya divergencia se demuestra crucial en el caso americano, se separan con menor intensidad en el caso español, y proponer que esta observación es coherente con la debilidad y con la poca profundidad del avance de la electrificación en nuestro país. Más concretamente, las comparaciones se basan fundamentalmente en datos sobre los precios relevantes para el conjunto de la economía española y de forma más minuciosa para el País Vasco. La elección del País Vasco para su estudio más detallado se justifica por su importancia relativa en el proceso industrializador español. En torno al cambio de siglo, la economía vasca se especializó en los sectores de industria pesada, transporte y navegación, todos ellos con gran capacidad de consumo de energía. Durante los años de la Segunda República el consumo vasco de carbón equivalía al 22 por 100 del total español'. A principios de la década de los años cuarenta el consumo de electricidad por habitante era cinco veces superior a la media nacional ${ }^{2}$. En cuanto a la distribución del consumo entre las tres provincias, Vizcaya ocupa una posición destacada respecto a las demás. En torno a 1930 le correspondía el 65 por 100 del consumo eléctrico y el 73 por 100 del de carbón. Alava es la que tenía el consumo más modesto, el 6 por 100 del consumo vasco de electricidad -y el 1,5 por 100 del carbón ${ }^{3}$.

1 Los únicos datos sobre distribución provincial del consumo de carbón disponibles son los de la Dirección General de Minas y Combustibles, citados en $\mathrm{S}$. Coll y C. Sudrià (1987), pp. 373-374.

2 Estimación de E. Uriarte (1945).

3 Respecto al consumo de electricidad, F. Antolín (1986 a). Para el carbón, S. Coll y C. Sudrià (1987), pp. 378-381. 


\section{Los datos}

La información esencial utilizada consiste, por una parte, en los precios de energías alternativas y demás factores productivos; $y$, por otra, indicadores relativos a la extensión e intensidad del proceso de electrificación. Comparando la evolución de los precios relativos en nuestro caso con la del caso americano se puede establecer la mayor o menor importancia de las ventajas de costes ofrecidas por la energía eléctrica respecto a otros factores de producción industrial. Comparando los grados de penetración de la electricidad se puede evaluar si existe o no correlación entre la intensidad de aquellos impulsos y la presunta respuesta adaptativa de la economía. Los datos efectivamente utilizados para la comparación con el caso americano se refieren al período 1913-29. La elección de este período viene impuesta por la oportunidad de comparar las cifras españolas con las de los estudios reseñados. Se trata indudablemente de un período crucial para la implantación definitiva de la electricidad, enmarcado entre la Primera Guerra Mundial y la depresión de la década de 1930. Sin embargo, en el Apéndice se proponen series más largas que abarcan para algunas variables desde 1901 hasta 1944. Esto me permite utilizar rasgos de la evolución de las magnitudes relevantes más allá del marco limitado en que se mueve la comparación internacional. Por otra parte, considero que los datos sobre precios de la energía disponibles para el caso vasco son especialmente ricos, y su sistematización para un periodo prolongado puede ser también, en sí misma, una de las aportaciones del trabajo.

He recogido información sobre costes de la electricidad, del carbón y salarios. Para evaluar la extensión e intensidad de la electrificación, presento datos sobre potencias instaladas, en energía hidráulica, vapor y electricidad para diversas industrias que son grandes consumidoras de energía; y también sobre la distribución de la potencia eléctrica instalada entre pequeños y grandes productores. La información esencial sobre costes en el País Vasco viene referida a la provincia de Vizcaya. Las informaciones adicionales se utilizan, sobre todo, para contrastar la plausibilidad de que las conclusiones aquí obtenidas se generalicen al caso vasco en particular, y al español en general.

En el Apéndice se presentan todos los datos con referencia a las fuentes, y se discute también su significación y los criterios utilizados para seleccionar los que son objeto de un análisis más detallado, y que reproduzco en el cuadro 1 . 


\section{CUADRO 1}

Precios del carbón, electricidad y salarios en el País Vasco y España (Pesetas corrientes)

\begin{tabular}{|c|c|c|c|c|c|c|}
\hline \multirow[b]{2}{*}{ Años } & \multicolumn{3}{|c|}{ PAIS VASCO } & \multicolumn{3}{|c|}{ ESPAÑ̄A } \\
\hline & $\begin{array}{l}\text { Carbón } \\
\text { asturiano }\end{array}$ & $\begin{array}{c}\text { Electricidad } \\
H E I\end{array}$ & Salarios & Carbón SC & $\begin{array}{c}\text { Carbón } \\
\text { importado }\end{array}$ & Electricidad \\
\hline $\begin{array}{lll}1913 & \ldots & \ldots \\
1914 & \ldots & \ldots \\
1915 & \ldots & \ldots \\
1916 & \ldots & \ldots \\
1917 & \ldots & \ldots \\
1918 & \ldots & \ldots \\
1919 & \ldots & \ldots \\
1920 & \ldots & \ldots \\
1921 & \ldots & \ldots \\
1922 & \ldots & \ldots \\
1923 & \ldots & \ldots \\
1924 & \ldots & \ldots \\
1925 & \ldots & \ldots \\
1926 & \ldots & \ldots \\
1927 & \ldots & \ldots \\
1928 & \ldots & \ldots \\
1929 & \ldots & \ldots\end{array}$ & $\begin{array}{l}26 \\
29 \\
36 \\
70,5 \\
125 \\
160 \\
125 \\
138 \\
97 \\
57 \\
57,5 \\
63,5 \\
56 \\
58 \\
54 \\
48 \\
51,25\end{array}$ & $\begin{array}{l}4 \\
4,19 \\
4,27 \\
4,81 \\
6,1 \\
6,54 \\
5,88 \\
7,09 \\
7,1 \\
7,3 \\
6,05 \\
6,27 \\
6,5 \\
6,5 \\
6,2 \\
6,45 \\
7,05\end{array}$ & $\begin{array}{l}3,25 \\
3,25 \\
3,25 \\
3,57 \\
3,85 \\
4,5 \\
5,5 \\
6,9 \\
6,9 \\
5,85 \\
5,85 \\
6,5 \\
7 \\
7 \\
7 \\
7 \\
7\end{array}$ & $\begin{array}{l}20 \\
21 \\
30 \\
57 \\
67 \\
66 \\
55 \\
63 \\
47 \\
35 \\
37 \\
39 \\
37 \\
36 \\
36 \\
36 \\
35\end{array}$ & $\begin{array}{r}34 \\
32 \\
52 \\
72 \\
166 \\
248 \\
125 \\
135 \\
75 \\
61 \\
62 \\
65 \\
58\end{array}$ & $\begin{array}{l}0,21 \\
0,28 \\
0,27 \\
0,26 \\
0,25 \\
0,23 \\
0,25 \\
0,24 \\
0,25 \\
0,25 \\
0,25 \\
0,24 \\
0,23 \\
0,24 \\
0,24 \\
0,24 \\
0,22\end{array}$ \\
\hline
\end{tabular}

FuEntes: País Vasco: carbón asturiano destinado a Bilbao (ptas. tonelada), $B$. $O$. de Estadística del Ayuntamiento de Bilbao; electricidad (ptas. kwh producido), Hidroeléctrica Ibérica; salario (ptas. jornal), R. Reina (1933). España: carbón español, precio medio en origen, y carbón importado, precio medio en el litoral español (ptas. tonelada), S. Coll y C. Sudrià (1987); electricidad (precio medio en kwh de la energía vendida para todos los usos), Anuario Estadístico de España.

Evolución de los precios del carbón, electricidad y salarios

La evolución de los precios en Bilbao-País Vasco y España se muestra en los gráficos 1 y 2. En el País Vasco el impacto de la Primera Guerra Mundial fue especialmente notable al ser ésta una zona que habitualmente se abastecía de carbón inglés. El crecimiento del precio del carbón asturiano destinado a Bilbao en el intervalo 1916-1921 es muy superior al del carbón español para el conjunto de España (gráfico 2), mientras que guarda una estrecha relación con el precio del carbón inglés en el litoral español, que aparece en el mismo gráfico 2. 


\section{CUADRO 2}

Evolución de los precios relativos en Bilbao-País Vasco (Indice)

\begin{tabular}{|c|c|c|c|c|c|c|c|c|}
\hline & $A \bar{n}$ & ios & & & & Carbón & Electricidad & Salarios \\
\hline $\begin{array}{ll}1913 & \ldots \\
1914 & \ldots \\
1915 & \ldots \\
1916 & \ldots \\
1917 & \ldots \\
1918 & \ldots \\
1919 & \ldots \\
1920 & \ldots \\
1921 & \ldots \\
1922 & \ldots \\
1923 & \ldots \\
1924 & \ldots \\
1925 & \ldots \\
1926 & \ldots \\
1927 & \ldots \\
1928 & \ldots \\
1929 & \ldots\end{array}$ & 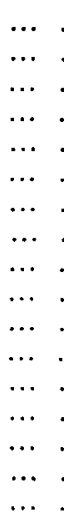 & $\begin{array}{l}\cdots \\
\cdots \\
\cdots \\
\cdots \\
\cdots \\
\cdots \\
\cdots \\
\cdots \\
\cdots \\
\cdots \\
\cdots \\
\cdots \\
\cdots \\
\cdots \\
\cdots \\
\cdots \\
\end{array}$ & $\begin{array}{l}\ldots \\
\ldots \\
\ldots \\
\ldots \\
\cdots \\
\cdots \\
\cdots \\
\cdots \\
\cdots \\
\cdots \\
\cdots \\
\cdots \\
\cdots \\
\cdots \\
\cdots \\
\ldots \\
\ldots\end{array}$ & $\begin{array}{l}\ldots \\
\ldots \\
\ldots \\
\ldots \\
\ldots \\
\ldots \\
\ldots \\
\ldots \\
\ldots \\
\cdots \\
\cdots \\
\cdots \\
\cdots \\
\cdots \\
\cdots \\
\ldots \\
\end{array}$ & $\begin{array}{l}\ldots \\
\ldots \\
\ldots \\
\ldots \\
\ldots \\
\ldots \\
\ldots \\
\ldots \\
\ldots \\
\ldots \\
\ldots \\
\ldots \\
\ldots \\
\ldots \\
\ldots \\
\ldots\end{array}$ & $\begin{array}{l}100 \\
111 \\
138 \\
271 \\
480 \\
615 \\
480 \\
530 \\
361 \\
219 \\
221 \\
244 \\
215 \\
223 \\
207 \\
184 \\
197\end{array}$ & $\begin{array}{l}100 \\
105 \\
107 \\
120 \\
152 \\
163 \\
147 \\
177 \\
177 \\
182 \\
151 \\
156 \\
162 \\
162 \\
155 \\
161 \\
176\end{array}$ & $\begin{array}{l}100 \\
100 \\
100 \\
110 \\
118 \\
138 \\
169 \\
212 \\
212 \\
180 \\
180 \\
200 \\
215 \\
215 \\
215 \\
215 \\
215\end{array}$ \\
\hline
\end{tabular}

FuENTE: Cuadro 1.

\section{GRAFICO 1}

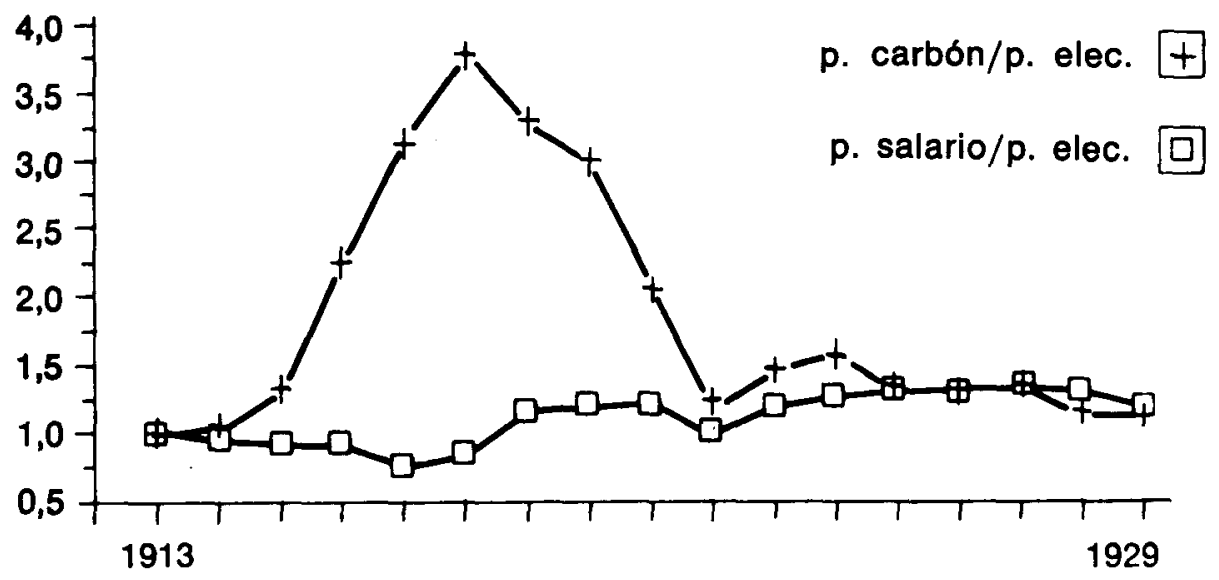

FUENTE: Cuadro 1. 


\section{CUADRO 3}

Evolución de los precios relativos en España. Indice

\begin{tabular}{|c|c|c|c|c|}
\hline & $\mathrm{AH}$ & AI & $\mathrm{AJ}$ & $\mathrm{AK}$ \\
\hline Años & Carbón (a) & Carbón (i) & Electricidad & Salarios \\
\hline $\begin{array}{lllllll}1913 & \ldots & \ldots & \ldots & \ldots & \ldots & \ldots \\
1914 & \ldots & \ldots & \ldots & \ldots & \ldots & \ldots \\
1915 & \ldots & \ldots & \ldots & \ldots & \ldots & \ldots \\
1916 & \ldots & \ldots & \ldots & \ldots & \ldots & \ldots \\
1917 & \ldots & \ldots & \ldots & \ldots & \ldots & \ldots \\
1918 & \ldots & \ldots & \ldots & \ldots & \ldots & \ldots \\
1919 & \ldots & \ldots & \ldots & \ldots & \ldots & \ldots \\
1920 & \ldots & \ldots & \ldots & \ldots & \ldots & \ldots \\
1921 & \ldots & \ldots & \ldots & \ldots & \ldots & \ldots \\
1922 & \ldots & \ldots & \ldots & \ldots & \ldots & \ldots \\
1923 & \ldots & \ldots & \ldots & \ldots & \ldots & \ldots \\
1924 & \ldots & \ldots & \ldots & \ldots & \ldots & \ldots \\
1925 & \ldots & \ldots & \ldots & \ldots & \ldots & \ldots \\
1926 & \ldots & \ldots & \ldots & \ldots & \ldots & \ldots \\
1927 & \ldots & \ldots & \ldots & \ldots & \ldots & \ldots \\
1928 & \ldots & \ldots & \ldots & \ldots & \ldots & \ldots \\
1929 & \ldots & \ldots & \ldots & \ldots & \ldots & \ldots \\
& \ldots & \ldots & \ldots\end{array}$ & $\begin{array}{l}100 \\
105 \\
150 \\
285 \\
335 \\
330 \\
275 \\
315 \\
235 \\
175 \\
185 \\
195 \\
185 \\
180 \\
180 \\
180 \\
175\end{array}$ & $\begin{array}{r}100 \\
94 \\
153 \\
213 \\
488 \\
729 \\
368 \\
397 \\
221 \\
179 \\
182 \\
191 \\
171\end{array}$ & $\begin{array}{l}100 \\
127 \\
123 \\
120 \\
114 \\
108 \\
114 \\
110 \\
114 \\
114 \\
114 \\
111 \\
107 \\
109 \\
108 \\
108 \\
104\end{array}$ & $\begin{array}{l}100 \\
103 \\
108 \\
120 \\
130 \\
169 \\
195 \\
237 \\
222 \\
205 \\
211 \\
216 \\
216 \\
213 \\
213 \\
224 \\
226\end{array}$ \\
\hline
\end{tabular}

Fuentes: Los índices del carbón y la electricidad los obtengo del cuadro 1, y el índice de salarios, de J. Maluquer (1988).

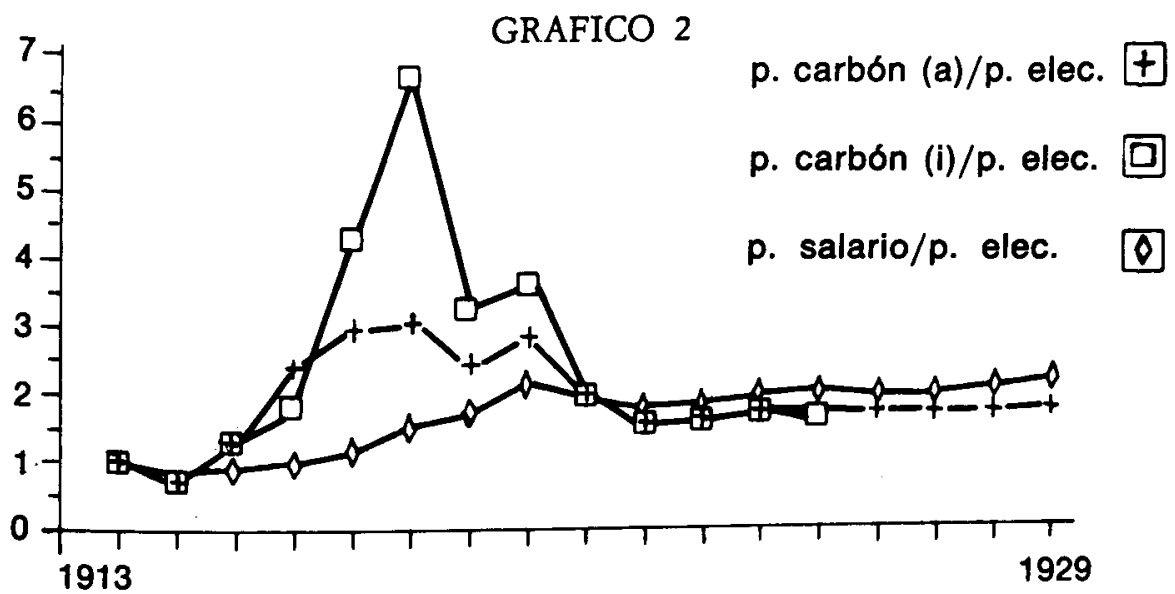

FUENTES: Los índices del carbón y la electricidad los obtengo del cuadro 1, y el índice de salarios, de J. Maluquer (1988). 
La explicación de dicho fenómeno reside en la fuerte dependencia del consumo bilbaíno respecto al carbón inglés y al comportamiento mimético que respecto a él tiene el carbón español. Transcurridos los seis años de fuerte subida de los precios del carbón en Bilbao, éstos se estabilizan a un nivel algo superior al del conjunto español.

El comportamiento de los precios de la electricidad, el carbón y el trabajo en España tiene ciertas similitudes con el descrito por Woolf respecto a la economía americana en el mismo período. Se produce un incremento en el precio del carbón y en el del trabajo, y si bien el precio de la electricidad no baja en términos absolutos, sí lo hace en términos relativos. Comparando el cambio en los precios relativos, vemos cuáles son las diferencias entre ambas trayectorias de precios.

\section{CUADRO 4}

Precios de varios «inputs» en el año $1929(1913=100)$

\begin{tabular}{|c|c|c|c|c|c|}
\hline & Carbón & Salarios & Electricidad & $\begin{array}{l}\text { Carbón/ } \\
\text { electricidad }\end{array}$ & $\begin{array}{l}\text { Salarios/ } \\
\text { electricidad }\end{array}$ \\
\hline EE. UU. ... . . . . . & 300 & 200 & 50 & 6 & 4 \\
\hline $\begin{array}{lllll}\text { España } & \ldots & \ldots & \ldots & \ldots\end{array}$ & 175 & 226 & 164 & 1,7 & 2,2 \\
\hline $\begin{array}{lllll}\text { Bilbao } & \ldots & \ldots & \ldots & \ldots\end{array}$ & 197 & 215 & 176 & 1,1 & 1,2 \\
\hline
\end{tabular}

Furntres: Cuadros 2 y 3.

Escogiendo unidades tales que para el año base de 1913 los precios relativos entre carbón, trabajo y electricidad fuesen todos iguales a uno, vemos cómo, en 1929, el precio del carbón había crecido en EE. UU. seis veces más, y el del trabajo cuatro veces más que el de la electricidad. En Bilbao, en cambio, las variaciones entre precios relativos, aunque en la misma dirección, son mucho menores. El del carbón crece un 1,2 y el del trabajo un 1,1 más que el de la electricidad. Para el total de España, el precio del carbón aumenta 1,7 veces más que el de la electricidad, y el del trabajo 2,2 . Si identificamos el mayor aumento relativo de los precios del carbón con respecto a la electricidad como un estimulo a la electrificación, y el mayor aumento relativo de los precios del trabajo con respecto a los de la electricidad como un estímulo a la capitalización, vemos que tanto uno como otro son mucho más débiles en el caso vasco, y también el conjunto español, que en los EE. UU. 
La comparación con los datos de Woolf me ha forzado a seleccionar el año 1929 para ponderar el distanciamiento en los precios relativos. Pero ¿Cuán significativo es este año? Como se ha visto en los gráficos 1 y 2 , la trayectoria de los precios es muy poco accidentada, a excepción de los años - seis en Bilbao y cinco en España- en que los precios del carbón se disparan como consecuencia de la Primera Guerra Mundial. ¿Pudieron estos años ser especialmente cruciales para abandonar el carbón y adoptar la electricidad? En el caso vasco y en el conjunto español, en vista de cuál fue su proceso de electrificación, parece que tuvieron mayor fuerza los factores desincentivadores. Durante la guerra se reduce el nivel de actividad de la economía vizcaína y por tanto su demanda de energía ${ }^{4}$. La subida de los precios del carbón estaba directamente relacionada con un hecho excepcional, y como se comprobó más tarde, éstos volvieron a sus cauces normales una vez concluida la guerra. Un motivo demasiado circunstancial para justificar un cambio de tecnología energética de la que, en parte, dependíamos todavía del exterior. No obstante, el movimiento de los precios relativos y el impacto de la guerra sobre la economía fue muy distinto en Cataluña. En esta zona, con un elevado consumo de energía por habitante, el carbón fue siempre más caro que en Bilbao y la electricidad más barata, porque se introdujo en condiciones de fuerte competencia. Durante los años de la guerra, la industria catalana se vio estimulada. En varios trabajos, J. Maluquer (1984 y 1987) muestra la aceleración que experimenta la producción de electricidad en el período de la guerra y la casi total sustitución del vapor por la electricidad en ciertos sectores de industria ligera, predominante en Cataluña.

\section{Electrificación}

La electrificación se inició en el País Vasco en la última década del siglo xIx y fue progresando al ritmo que muestra el cuadro 5 . En ella he diferenciado la capacidad de las instalaciones de la empresa que la produce y distribuye a gran escala - Hidroeléctrica Ibérica (HEI) - del resto de compañias eléctricas de menor entidad y autoproductores (otras).

- Según las memorias de la sociedad Hidroeléctrica Ibérica, durante los años de la guerra europea descendió el volumen de sus ventas de energía. Dicho fenómeno se aprecia también en la evolución del índice de producción industrial vasca; véase A. Carreras (1985). 


\section{CUADRO 5}

Potencia en kw de las instalaciones eléctricas que surten el Pais Vasco

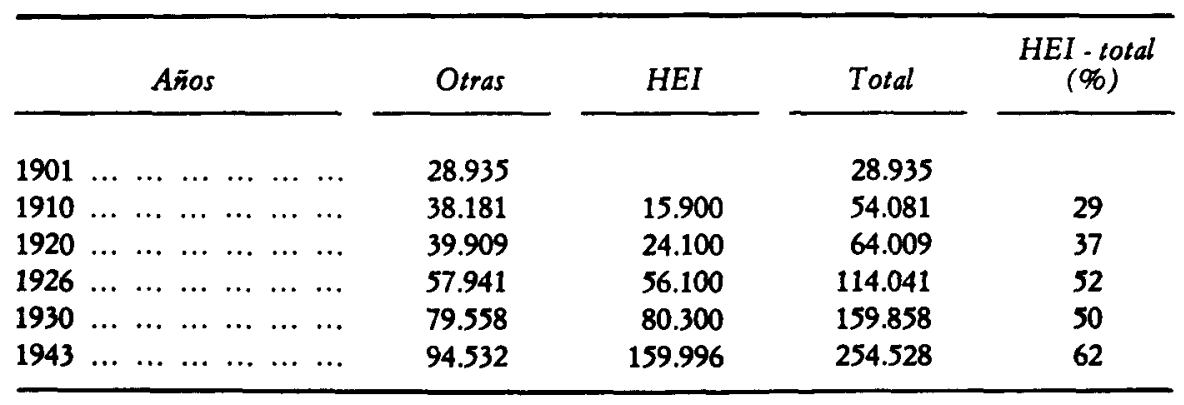

FuENTE: F. Antolin (1988).

En 1920, año en que los grandes productores americanos, los únicos capaces de capturar las economías de escala en esta industria, surtían el 57 por 100 de sus mercados, en el País Vasco HEI cubría tan sólo el 37 por 100 del mercado. Finalmente, en el año 1943, la potencia de producción de HEI ya es mayoritaria: supone el 62 por 100 de la de pequeños productores. Así, pues, sea cual sea el grado de implantación de la electricidad como fuente de energía, está claro que, en el caso vasco, muchas de las potenciales ventajas de costes asociadas con rendimientos a escala no van a ser aprovechadas.

Mucho más difícil de documentar es la adopción de la nueva tecnología por parte de las empresas: la transición del vapor a la electricidad. Las tablas siguientes recogen experiencias parciales. El cuadro 6 muestra la potencia instalada por ambos conceptos en las empresas del metal más importantes en Vizcaya. En él destaca la tardanza en la introducción de la electricidad, y su modesta contribución frente al vapor. Los cuadros 7 y 8 muestran la potencia instalada en empresas del metal y cemento en Guipúzcoa, y metal y asfalto en Alava, respectivamente. En Alava, donde el transporte encarece extraordinariamente el carbón, la electricidad es en las industrias documentadas preponderante desde 1910. En Guipúzcoa, no obstante, se repite el mismo comportamiento que en Vizcaya. En 1920, cuando electricidad es mayoritaria en la economía americana, en las industrias vascas más significativas representa tan sólo el 15 por 100 (Vizcaya) y el 31 por 100 (Guipúzcoa) de la potencia instalada.

Lo que sabemos respecto al comportamiento del sector energético e industrial español permite concluir que en nuestro pasado industrial no se da 


\section{CUADRO 6}

Potencia (CV) de máquinas bidráulicas, de vapor y eléctricas en grandes empresas del metal en Vizcaya

\begin{tabular}{|c|c|c|c|c|c|c|c|}
\hline A & B & C & D & $\mathbf{E}$ & F & G & $\mathbf{H}$ \\
\hline$A \tilde{o} o s$ & $\begin{array}{c}N .^{\circ} \text { de } \\
\text { empresas }\end{array}$ & $\begin{array}{l}\text { Hidráu- } \\
\quad \text { lica }\end{array}$ & Vapor & $\begin{array}{l}\text { Eléc- } \\
\text { trica }\end{array}$ & $\begin{array}{l}\text { Eléc- } \\
\text { trica * }\end{array}$ & $\begin{array}{c}\text { Total } \\
(\mathrm{D}+\mathrm{F})\end{array}$ & $\begin{array}{c}\text { Eléctrica } \\
(\%)\end{array}$ \\
\hline $1900 \ldots \ldots$ & 5 & 310 & 22.237 & & & 22.237 & \\
\hline $1910 \ldots \ldots$ & 4 & 280 & 23.535 & 87 & 17 & 23.552 & \\
\hline $1920 \ldots \ldots$ & 6 & 1.020 & 39.133 & 34.969 & 6.993 & 46.126 & 15 \\
\hline $1926 \ldots \ldots$ & 4 & 1.818 & 27.066 & 35.314 & 7.063 & 34.139 & 20 \\
\hline $1930 \ldots \ldots$ & 6 & 1.950 & 44.589 & 105.279 & 21.060 & 65.649 & 32 \\
\hline $1934 \ldots \ldots$ & & & 93.598 & 109.126 & 21.825 & 115.423 & 28 \\
\hline $1939 \ldots \ldots$ & 2 & & 54.597 & 110.048 & 22.010 & 76.607 & 29 \\
\hline
\end{tabular}

* Las cifras de la columna $F$ del cuadro 6 y E de los cuadros 7 y 8 se obtienen dividiendo la potencia de las máquinas eléctricas por cinco. Según Devine, de la potencia eléctrica instalada, sólo un quinto puede considerarse activa a objeto de comparación con el vapor.

Fuente: Estadistica Minera.

\section{CUADRO 7}

Potencia $(C V)$ de máquinas bidráulicas, de vapor y eléctricas en grandes empresas del metal y cemento en Guipuizcoa

\begin{tabular}{|c|c|c|c|c|c|c|}
\hline A & B & $C$ & D & $\mathbf{E}$ & $\mathbf{F}$ & $\mathbf{G}$ \\
\hline Años & $\begin{array}{c}\text { Hidráu- } \\
\text { lica }\end{array}$ & Vapor & $\begin{array}{l}\text { Eléc- } \\
\text { trica }\end{array}$ & $\begin{array}{l}\text { Eléc- } \\
\text { trica }\end{array}$ & $\begin{array}{c}\text { Total } \\
(\mathrm{C}+\mathrm{E})\end{array}$ & $\begin{array}{c}\text { Electrica } \\
(\%)\end{array}$ \\
\hline $1902 \ldots \ldots$ & 880 & 2.396 & & & 2.390 & \\
\hline $1910 \ldots \ldots$ & 909 & 1.105 & 1.111 & 222 & 1.327 & 16 \\
\hline $1920 \ldots \ldots$ & 424 & 1.120 & 2.580 & 516 & 1.636 & 31 \\
\hline $1930 \ldots \ldots$ & 3.709 & 3.640 & 9.130 & 1.826 & 5.466 & 33 \\
\hline $1933 \ldots \ldots$ & & 2.000 & 8.857 & 1.771 & 3.771 & 47 \\
\hline
\end{tabular}

FusNre: Estadistica Minera. 


\section{CUADRO 8}

Potencia $(\mathrm{CV})$ de máquinas bidráulicas, de vapor y eléctricas en grandes empresas del metal y asfalto en Alava

\begin{tabular}{|c|c|c|c|c|c|c|}
\hline$A$ & B & C & D & $\mathrm{E}$ & $\mathrm{F}$ & $G$ \\
\hline$A \bar{x} o s$ & $\begin{array}{c}\text { Hidráu- } \\
\text { lica }\end{array}$ & Vapor & $\begin{array}{l}\text { Eléc- } \\
\text { trica }\end{array}$ & $\begin{array}{l}\text { Elèc- } \\
\text { trica }\end{array}$ & $\begin{array}{c}\text { Total } \\
(\mathrm{C}+\mathrm{E})\end{array}$ & $\begin{array}{c}\text { Eléctrica } \\
(\%)\end{array}$ \\
\hline $\begin{array}{lll}1902 & \ldots & \ldots \\
1910 & \ldots & \ldots \\
1920 & \ldots & \ldots \\
1930 & \ldots & \ldots \\
1933 & \ldots & \ldots\end{array}$ & $\begin{array}{r}282 \\
600 \\
200 \\
30\end{array}$ & $\begin{array}{r}60 \\
30 \\
25 \\
5\end{array}$ & $\begin{array}{r}420 \\
550 \\
1.072 \\
6.753\end{array}$ & $\begin{array}{r}84 \\
110 \\
214 \\
1.350\end{array}$ & $\begin{array}{r}60 \\
114 \\
135 \\
214 \\
1.355\end{array}$ & $\begin{array}{r}73 \\
81 \\
100 \\
100\end{array}$ \\
\hline
\end{tabular}

FueNTE: Estadistica Minera.

la conexión apuntada por Devine entre electrificación y crecimiento económico. Mientras que en EE. UU. la adopción generalizada de la electricidad es simultánea al ahorro de energía por unidad del PNB y al incremento en la productividad del trabajo y del capital, Sudrià (1987) observa que en el caso español la ratio consumo de energía/PNB es siempre creciente. Y Carreras (1987) estima un descenso en la productividad del trabajo industrial en las primeras décadas del siglo. La productividad del trabajo no remonta el nivel de 1900 hasta la década de 1960 , mientras que, en el conjunto español, la electricidad seguramente superaría al vapor una vez finalizada la Guerra Civil. En la posguerra se regula el sector eléctrico, congelándose efectivamente los precios de la electricidad al tiempo que aumenta el precio de los demás inputs energéticos, dadas las escaseces del período y la dificultad de controlar ciertos mercados por parte de la Administración.

En España parece cumplirse mejor la hipótesis de Woolf. Son las ventajas de precios relativos entre electricidad, carbón y trabajo, más que las puramente tecnológicas, las que activan el proceso de electrificación y abren las puertas a una mayor capitalización de la economía industrial. $Y$, en nuestro caso, a un distanciamiento muy modesto entre precios relativos le corresponde un proceso de avance de la electrificación muy lento respecto al vapor $y$ poco radical en cuanto a la estructura interna del sector productor ${ }^{5}$.

' En Vizcaya y Guipúzcoa, HEI, la empresa capaz de producir más eficientemente y, en consecuencia, de vender a menor precio, no consigue desplazar a los productores marginales. En un trabajo anterior he documentado la explotación de los recursos hidráulicos en la cuenca del Bajo Nervión (Vizcaya). En él observo que dicha explotación se mantuvo 
Este trabajo se ha centrado en el análisis de precios relativos, al tratar de entender un proceso de sustitución de factores. Pero nuestros datos abundan también en un tema ya destacado por otros autores, el de la carestía de la energía en España en términos absolutos. En efecto, los estudios realizados sobre el coste del carbón disponible para la industria española demuestran que el vapor fue una energía cara en relación a otros países ( $\mathrm{Na}$ dal et al., 1983). Siendo el carbón ya caro para empezar, y el distanciamiento conseguido entre los precios de éste y los de la electricidad mucho menor que en la economía americana, hay que concluir que el coste de la electricidad en España en relación a otros paises compara de manera mucho más desfa. vorable de lo que compara el vapor.

\section{Apéndice}

La información más exhaustiva sobre precios de la energía es la que facilita el Boletín Oficial de Estadistica del Ayuntamiento de Bilbao (EAB) desde 1913 en adelante. Este incluye los precios de detallista y mayorista de los principales inputs energéticos que surten el consumo de Bilbao. Los precios de detallista son por definición los que reproducen con mayor fidelidad el coste del consumo de combustible, aunque no siempre convenga utilizarlos. Como es normal, tienen una oscilación más acentuada que los precios de mayorista, puesto que recogen la presión de la demanda ante carestías y expectativas puntuales. De la comparación de los precios de mayorista de la estadística del Ayuntamiento de Bilbao con la de las otras dos fuentes reproducidas se observa que los precios del Ayuntamiento son casi siempre más altos. Posiblemente se deba a que la información no proviene de los productores de carbón, lo cual los hace más realistas, puesto que a éstos se les atribuye un sesgo a la baja. En el cuadro A-2 incluyo también un indicador del precio de la electricidad surtida por Hidroeléctrica Ibérica (HEI), la productora más importante del País Vasco, así como el precio del jornal minero, que obtengo de otra fuente, más completa que la del Ayuntamiento, pero en la que no observo discrepancias en los años en que tengo información de ambas. Los datos para el conjunto español sobre carbón y electricidad los obtengo del Anuario Estadistico de España (AEE), si bien para el carbón existe la estimación más completa y rigurosa de S. Coll y C. Sudrià (1987).

Electricidad. Los precios de la electricidad para consumo doméstico en Bilbao (1913-1944) [cuadro A-1, col. G] pueden completarse con los de las

y progresó posteriormente a que HEI se convirtiera en el mayor productor vasco de electricidad, y que superó la magnitud de los recursos hidráulicos disponibles considerada económicamente rentable. Véase F. Antolín (1986). 
estadísticas oficiales de principios de siglo ${ }^{\circ}$. En 1901, el precio del kWh para iluminación privada era de 0,90 pts. en Bilbao (la Electra Compañía General de Electricidad) y poblaciones industriales de la Ría (Electra del Nervión [Baracaldo] y Santa Ana de Bolueta [Begoña]), y algo más barato en el resto de poblaciones vizcaínas que tenían suministro (aprox. $0,70 \mathrm{pts} . \mathrm{kWh}$ ). Seguramente los precios bajaron cuando HEI inició el suministro a la Ría en 1904, porque, a pesar de la subida de un 26 por 100 entre 1913 y 1921 , en 1926, cuando de nuevo tenemos datos, el precio del $\mathrm{kWh}$ era de $0,62 \mathrm{pts}$. (col. H). Para seguir la evolución de los precios de la electricidad en Bilbao no queda más remedio que utilizar la serie de remuneración media del $\mathrm{kWh}$ producido por HEI (cuadro A-2, col. F), puesto que las tarifas domésticas del cuadro A-1, col. G, se refieren a magnitudes distintas en los distintos tramos de la serie ${ }^{7}$. La electricidad en San Sebastián tiene, a principio de siglo (estadísticas oficiales), un precio algo inferior al de Bilbao, 0,80 pts. el $\mathrm{kWh}$ (Compañía Eléctrica de San Sebastián). En Vitoria, el precio es de 0,75 pts. el $\mathrm{kWh}$ (Electra Vitoriana) y, al igual que en Vizcaya, el precio en las poblaciones más importantes de estas dos provincias vascas es algo inferior al de la capital. Según la estadística de 1920 , al cabo de veinte años los márgenes de precios marcados a principio de siglo, entre las capitales vascas y entre éstas y sus entornos, parecen mantenerse, en lo que se refiere a pequeños suministradores locales. A partir de entonces la porción del mercado surtido por HEI fue en Guipúzcoa y Vitoria cada vez mayor, por lo que los precios, con un margen de diferencia a principio de siglo de por sí insignificante, se irían unificando con los de Vizcaya. Cabe suponer por

- Estadística de la Industria Eléctrica en España (años 1901, 1905, 1910), Ministerio de Agricultura, Industria, Comercio y Obras Públicas, Madrid.

1 La magnitud de la ratio remuneración media por $\mathrm{kwh}$ producido viene determinada por la amalgama de tarifas y tipos de contrato suscritos por el conjunto de abonados, que para HEI son grandes empresas y distribuidores locales. Dicho indicador tiene, a mi entender, ventajas sobre la serie de tarifas domésticas, porque el consumidor doméstico es minoritario en términos de consumo relativo y porque el precio que éste paga es el más controlado por la administración local de toda la gama de precios que ofrecen las eléctricas. La rigidez de las tarifas domésticas respecto a los ingresos medios se observa con toda claridad en las series de precios presentadas en J. Maluquer (1987). En el tramo en que baja la remuneración media de cinco empresas eléctricas españolas, la tarifa do. méstica de Barcelona se sitúa por encima, y a la inversa cuando sube el indicador de remuneración. Otra constatación interesante de la comparación entre los dos tipos de series que reproduce Maluquer es que a lo largo del período éstas se distancian escasamente, lo cual me da confianza en la utilización de la única serie de que dispongo para el País Vasco. El precio de la electricidad de Woolf viene dado en tarifas domésticas. Según este autor, este hecho determina que sus precios de electricidad infravaloren el distanciamiento que se produce entre el precio de ésta, que se mueve a la baja, y el del carbón y salarios, que van al alza. Dado que en mi estudio no utilizo tarifas domésticas, el distanciamiento real entre precios relativos en los EE. UU. y en España es incluso mayor. 
tanto que el precio de la electricidad de $\mathrm{HEI}$ es un indicador válido para el conjunto vasco.

Carbón. El precio del carbón que utilizo para el cálculo de precios relativo en Bilbao es el del cribado asturiano de mayorista destinado a Bilbao. Las opciones rechazadas son: $a$ ) la de precios de detallista (cuadro A-1, col. E) porque éstos son excesivamente sensibles a expectativas y carestías muy puntuales que, a mi entender, son poco relevantes para la toma de decisiones sobre el cambio de tecnología energética, y b) la del carbón inglés, porque no tengo datos para los últimos años del período. La información sobre el coste de transporte del carbón a las capitales de provincia, para el período de la Segunda República, permite conocer la variación en el precio del carbón en las capitales vascas ${ }^{8}$. En San Sebastián el carbón es tan sólo un 4 por 100 más caro que en Bilbao, mientras que en Vitoria la diferencia es de un 25 por 100 . Si suponemos que el precio del transporte no se alteró a lo largo del período, lo cual es muy plausible, puesto que en el intervalo de despegue en el precio (1918-1921) se intervino el precio del carbón destinado al transporte terrestre y marítimo, la diferencia de precio entre capitales, supuesta constante, altera la posición relativa del carbón respecto a la electricidad, que como veíamos tiene un mismo precio en el conjunto vasco, pero no el índice de precios del carbón, que depende de las variaciones del precio de éste a boca mina y que es común para toda el área. El índice de precios del carbón tomado para Bilbao es por tanto extensible al resto de capitales vascas.

' S. Coll y C. Sudrià (1987), pp. 452-469. 


\section{CUADRO A-1}

Principales «inputs» energéticos en Bilbao

(Precios de detallista)

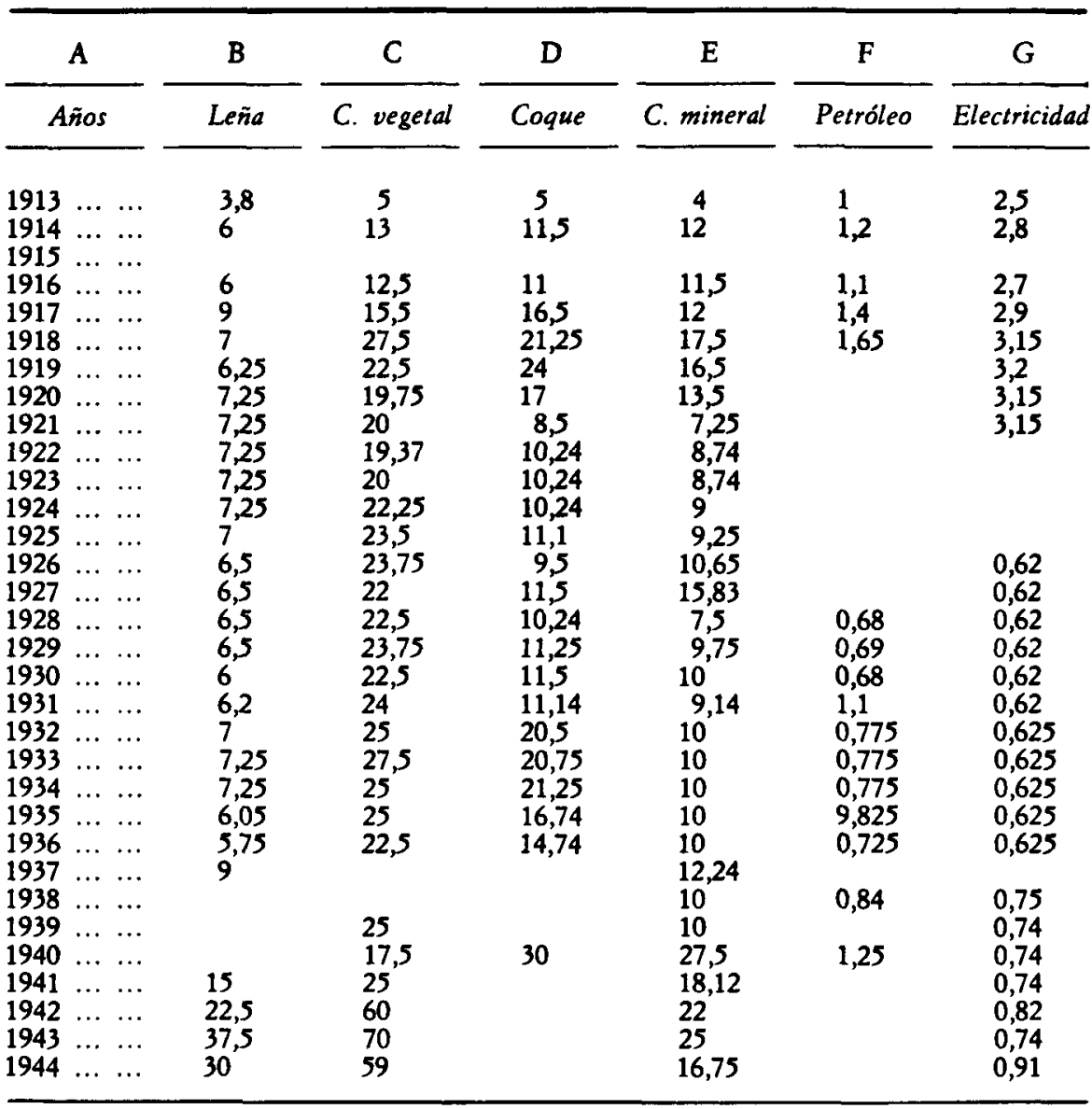

Unidades: leña, c. vegetal, c. mineral y coque (ptas. $100 \mathrm{~K}_{\mathbf{g}}$ ); petróleo (ptas. litro); gas (ptas. metro cúbico); electricidad (1913-1921, ptas. mes-5 bujías; 1926-1944, pesetas kwh).

FUENTE: Boletin Oficial de Estadistica del Ayuntamiento de Bilbao. 


\section{CUADRO A-2}

Carbón, electricidad y salarios en Bilbao

(Precios de mayorista)

\begin{tabular}{|c|c|c|c|c|c|c|}
\hline A & B & C & D & $\mathbf{E}$ & F & G \\
\hline$A \bar{n} o s$ & $\begin{array}{c}\text { C. asturiano } \\
E A B\end{array}$ & $\begin{array}{l}\text { C. inglés } \\
A E B\end{array}$ & $\begin{array}{l}\text { C. asturiano } \\
B A E E\end{array}$ & $\begin{array}{l}\text { C. inglés } \\
\text { BSC }\end{array}$ & $\begin{array}{c}\text { Electricidad } \\
H E I\end{array}$ & Salarios \\
\hline $\begin{array}{lll}1913 & \ldots & \ldots \\
1914 & \ldots & \ldots \\
1915 & \ldots & \ldots \\
1916 & \ldots & \ldots \\
1917 & \ldots & \ldots \\
1918 & \ldots & \ldots \\
1919 & \ldots & \ldots \\
1920 & \ldots & \ldots \\
1921 & \ldots & \ldots \\
1922 & \ldots & \ldots \\
1923 & \ldots & \ldots \\
1924 & \ldots & \ldots \\
1925 & \ldots & \ldots \\
1926 & \ldots & \ldots \\
1927 & \ldots & \ldots \\
1928 & \ldots & \ldots \\
1929 & \ldots & \ldots \\
1930 & \ldots & \ldots \\
1931 & \ldots & \ldots \\
1932 & \ldots & \ldots \\
1933 & \ldots & \ldots \\
1934 & \ldots & \ldots \\
1935 & \ldots & \ldots \\
1936 & \ldots & \ldots \\
1937 & \ldots & \ldots \\
1938 & \ldots & \ldots \\
1939 & \ldots & \ldots \\
1940 & \ldots & \ldots \\
1941 & \ldots & \ldots \\
1942 & \ldots & \ldots \\
1943 & \ldots & \ldots \\
1944 & \ldots & \ldots\end{array}$ & $\begin{array}{l}26 \\
29 \\
36 \\
70,5 \\
125 \\
160 \\
125 \\
138 \\
94 \\
57 \\
57,5 \\
63,5 \\
56 \\
58 \\
54 \\
48 \\
51,25 \\
52 \\
53,62 \\
55,75 \\
57,625 \\
59,135 \\
58,37 \\
58,37 \\
58,15 \\
58,15 \\
58,35 \\
61,25 \\
61,25 \\
83 \\
83 \\
83,5\end{array}$ & $\begin{array}{c}30,65 \\
28 \\
42 \\
82,38 \\
136,28 \\
214,4 \\
149,21 \\
181,93 \\
73,93 \\
56,18 \\
70,21 \\
68,55 \\
60,17\end{array}$ & $\begin{array}{r}29 \\
32 \\
37 \\
78 \\
108 \\
169 \\
115 \\
137 \\
80 \\
56 \\
59 \\
60 \\
56 \\
71 \\
54 \\
50 \\
51 \\
52\end{array}$ & $\begin{array}{r}31 \\
29 \\
43 \\
72 \\
135 \\
200 \\
116 \\
133 \\
69 \\
55 \\
57 \\
61 \\
55\end{array}$ & $\begin{array}{c}4 \\
4,19 \\
4,27 \\
4,81 \\
6,1 \\
6,54 \\
5,88 \\
7,09 \\
7,1 \\
7,3 \\
6,05 \\
6,27 \\
6,5 \\
6,5 \\
6,2 \\
6,45 \\
7,05 \\
6,85 \\
6,9 \\
6,65 \\
6,7 \\
9,3 \\
8,75 \\
9,1 \\
10,8 \\
9,7 \\
8,65 \\
8,3 \\
8,6 \\
9,35 \\
9,05 \\
11,5\end{array}$ & $\begin{array}{l}3,25 \\
3,25 \\
3,25 \\
3,57 \\
3,85 \\
4,5 \\
5,5 \\
6,9 \\
6,9 \\
5,85 \\
5,85 \\
6,5 \\
7 \\
7 \\
7 \\
7 \\
7 \\
7,5 \\
7,5 \\
7,5\end{array}$ \\
\hline
\end{tabular}

Unidades: carbón (ptas. tonelada); electricidad (ptas. kwh); salarios (ptas. jornal).

Fuentes: Columnas B y C (carbón asturiano e inglés destinado a Bilbao), Boletín Oficial de Estadistica del Ayuntamiento de Bilbao; columna $\mathrm{D}$ (carbón asturiano destinado a Bilbao), Anuario Estadístico de España; columna E (carbón inglés en Bilbao), S. Coll y C. Sudrià (1987); columna F, E. Uriarte (1968); columna G, R. Reina (1933). 


\section{CUADRO A-3}

\section{Carbón y electricidad en España}

(Precios de mayorista)

\begin{tabular}{|c|c|c|c|c|c|c|c|c|c|}
\hline \multicolumn{7}{|c|}{$\mathbf{A}$} & \multirow{2}{*}{ 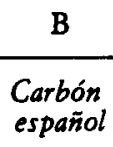 } & \multirow{2}{*}{$\frac{\mathrm{C}}{\underset{\text { Carbón }}{\text { importado }}}$} & \multirow{2}{*}{$\frac{\mathrm{D}}{\text { Electricidad }}$} \\
\hline & & & ños & & & & & & \\
\hline $\begin{array}{l}1913 \\
1914 \\
1915 \\
1916 \\
1917 \\
1918 \\
1919 \\
1920 \\
1921 \\
1922 \\
1923 \\
1924 \\
1925 \\
1926 \\
1927 \\
1928 \\
1929\end{array}$ & $\begin{array}{c}\ldots \\
\ldots \\
\ldots \\
\ldots \\
\ldots \\
\ldots \\
\ldots \\
\ldots \\
\ldots \\
\ldots \\
\ldots \\
\ldots \\
\ldots \\
\ldots \\
\ldots \\
\ldots\end{array}$ & $\begin{array}{l}\ldots \\
\ldots \\
\ldots \\
\ldots \\
\ldots \\
\ldots \\
\ldots \\
\ldots \\
\cdots \\
\cdots \\
\cdots \\
\cdots \\
\cdots \\
\cdots \\
\ldots\end{array}$ & $\begin{array}{l}\ldots \\
\ldots \\
\ldots \\
\ldots \\
\ldots \\
\ldots \\
\ldots \\
\ldots \\
\ldots \\
\ldots \\
\ldots \\
\ldots \\
\ldots \\
\ldots \\
\ldots \\
\ldots\end{array}$ & $\begin{array}{c}\ldots \\
\ldots \\
\ldots \\
\ldots \\
\ldots \\
\ldots \\
\ldots \\
\ldots \\
\ldots \\
\ldots \\
\ldots \\
\ldots \\
\ldots \\
\ldots \\
\ldots \\
\ldots\end{array}$ & $\begin{array}{c}\ldots \\
\ldots \\
\ldots \\
\ldots \\
\ldots \\
\ldots \\
\ldots \\
\ldots \\
\ldots \\
\ldots \\
\ldots \\
\ldots \\
\ldots \\
\ldots \\
\ldots\end{array}$ & $\begin{array}{c}\ldots \\
\ldots \\
\ldots \\
\ldots \\
\ldots \\
\ldots \\
\ldots \\
\ldots \\
\ldots \\
\ldots \\
\ldots \\
\ldots \\
\ldots \\
\ldots \\
\ldots \\
\ldots\end{array}$ & $\begin{array}{l}20 \\
21 \\
30 \\
57 \\
67 \\
66 \\
55 \\
63 \\
47 \\
35 \\
37 \\
39 \\
37 \\
36 \\
36 \\
36 \\
35\end{array}$ & $\begin{array}{r}34 \\
32 \\
52 \\
72 \\
166 \\
248 \\
125 \\
135 \\
75 \\
61 \\
62 \\
65 \\
58\end{array}$ & $\begin{array}{l}0,21 \\
0,28 \\
0,27 \\
0,26 \\
0,25 \\
0,23 \\
0,25 \\
0,24 \\
0,25 \\
0,25 \\
0,25 \\
0,24 \\
0,23 \\
0,24 \\
0,24 \\
0,24 \\
0,22\end{array}$ \\
\hline
\end{tabular}

Unidades: carbón (ptas. tonelada); electricidad (ptas. kwh).

FueNtes: Columnas B y C (carbón español, precio medio en origen, y carbón importado, precio medio en el litoral español), S. Coll y C. Sudrià (1987); columna D (precio medio de la energía vendida para todos los usos), Anuario Estadístico de España. 


\section{BIBLIOGRAFIA}

ANTolín, F. (1986): «Energía e industrialización en la cuenca del Bajo Nervión (18801930): La explotación tradicional de la energía hidráulica», en La industrialización del Norte de España, Ed. Crítica, Barcelona (en prensa).

- (1986 a): «Hidroeléctrica Ibérica-Iberduero. Un análisis de datos», IX Simposio de Análisis Económico, Universidad Autónoma de Barcelona.

- (1988): «Hidroeléctrica Ibérica y la electrificación del País Vasco» (trabajo mecanografiado sin publicar).

CARreras, A. (1985): «La producción industrial catalana y vasca. Los elementos para una comparación», en M. Gonzélez Portilla, J. Maluquer y B. DE Riquer (eds.), Industrialización y nacionalismo: análisis comparativos, Universidad Autónoma de Barcelona, Bellaterra.

- (1987): «La industria: atraso y modernización», en J. Nadal, A. Carreras y C. SuDRIX (eds.), La economia española en el siglo XX, Áriel.

Coll, S., y Sudrià, C. (1987): El carbón en España, 1770-1961, Turner, Madrid.

Devine, W. D., Jr. (1983): «From Shafts to Wires: Historical Perspective on Electrification», Journal of Economic History, 43, núm. 2.

Hrcks, J. (1932): The Theory of Wages, MacMillan, Londres.

Landes, D. (1969): The Unbound Prometheus, Cambridge Univ. Press, Cambridge (Mass.).

MaluQuer, J. (1983): «L'Electricitat", en J. Nadal et al. (1983).

- (1987): «L'Electricité, facteur de développement en Espagne, 1900-1936", en F. CarDOT (ed.), Un Siècle d'électricité dans le monde, 1880-1980, Presses Universitaires de France.

- (1988): «Precios, salarios y beneficios. La distribución funcional de la renta», en Estadísticas bistóricas de España, siglos XIX y XX, Fundación Banco Exterior, Madrid.

NADAL, J. (1986): «La debilidad de la industria química española en el siglo XIX. Un problema de demandam, Moneda y Crédito, núm. 176.

Nadal, J., et al. (1983): Producció $i$ consum d'energia en el creixement económic modern: el cas catalá, Barcelona.

Marshall, R. M. (1982): «Factors Influencing Changes in Productivity», en Ch. P. KINDLEBERGer y Guido do Tella (eds.), Economics in the Long View, The MacMillan Press.

Reina Y Cerezo, R. (1983): «La minería del hierro en Vizcayaw, Revista Minera.

Sudrì, C. (1987): «Un factor determinante: la energía», en J. Nadal, A. Carreras y C. SUDRIA (eds.), La economía española en el siglo XX, Ariel.

URIARTE, E. (1945): «La industria eléctrica en Vizcaya», en Primer Consejo Provincial de Ordenación Económica de Vizcaya.

- (1968): «Datos para la historia de Hidroeléctrica Ibérica" (trabajo mecanografiado sin publicar).

Woolf, A. G. (1984): «Electricity, Productivity, and Labor Saving: American Manufacturing, 1900-1929», Explorations in Economic History, vol. 2, núm. 2. 